\title{
Medicina de hospital y práctica del hospitalismo: una necesidad para el ejercicio actual de la medicina
}

\author{
Hospital medicine and hospitalism practice: a necessity \\ for the current practice of medicine
}

\author{
Moisés Auron, * Raúl Ariza Andraca ${ }^{\ddagger}$
}

\begin{abstract}
El hospital es la única escuela para crear a un verdadero discípulo de Esculapio. Abernethy en Aequanimitas de Williams Osler
\end{abstract}

La medicina suele ser una profesión en constante evolución y desarrollo que es impactada de manera continua por la influencia compleja de diversas vicisitudes (psicosociales, científicas, económicas, administrativas, etcétera). Además, en los últimos años, la complejidad de la práctica médica se ha incrementado notablemente debido a múltiples factores: el vertiginoso incremento y abundancia de la información, la exigencia de los enfermos, el escrutinio de los errores médicos y los crecientes y altos costos de la atención médica en todos los sentidos. Como una de las estrategias enfocadas para abordar estos problemas, se ha introducido la figura del llamado "médico hospitalista", un experto en los problemas médicos que se presentan en un hospital, por ejemplo, en la medicina perioperatoria, medicina trasfusional, urgencias hospitalarias, neumonía nosocomial, tromboembolia pulmonar, dolor, infecciones nosocomiales, daños por iatrogenia, COVID-19 o gestión hospitalaria. Así, el perfil ideal para ser hospitalista lo es el médico especialista con enfoque generalista (internista, intensivista, infectólogo, pediatra).

Pero ien qué consiste la medicina de hospital? Precisamente, la finalidad de este texto es comentar las bases y elementos fundamentales de la medicina de hospital.

* Departamentos de Medicina Hospitalaria de Adultos y Pediátrica, Cleveland Clinic. Cleveland, Ohio, Estados Unidos.

‡ Medicina Interna, Hospital Ángeles Pedregal, Ciudad de México.

Correspondencia:

Dr. Moisés Auron

Correo electrónico: auronm@ccf.org

Aceptado: 20-04-2020.

www.medigraphic.com/actamedica
Sin embargo, no pretender ser un documento exhaustivo ni específico, por lo que para un análisis más detallado, sugerimos, recurrir a fuentes más especializadas. El entrenamiento clásico del médico internista en la mayoría de los países ha considerado el desempeño profesional en múltiples ámbitos: la medicina de primer contacto y de consulta externa, urgencias y la práctica clínica en el paciente hospitalizado. En la medida del progreso profesional del médico y con la consiguiente demanda en crecimiento de la práctica clínica en ámbitos simultáneos, surge el reto de poder desempeñar una adecuada atención del paciente sin sacrificar la calidad de ésta.

La presencia de múltiples responsabilidades profesionales, incluyendo la práctica en el medio asistencial, aunada a la medicina privada (tal como ocurre en muchos países), genera una presión económica y emocional significativa, ya que, idealmente, el médico trabaja para vivir y disfruta ayudar al prójimo, y no de manera opuesta: el vivir para trabajar, lo cual ha derivado en el desarrollo y visibilización reciente de un fenómeno que, aunque lleva décadas, nadie se atrevía a señalar: el síndrome de fatiga o Burnout. Este síndrome impacta en múltiples víctimas (el médico, su familia y en la sociedad), representada por sus pacientes. Esto se ha evidenciado aún más al perder cierta independencia sobre el control de muchas variables: independencia clínica (la cual se ve influenciada por la necesidad de seguimiento de lineamientos y protocolos que parecieran arbitrarios en muchas ocasiones), la remuneración económica (la cual está controlada por sistemas de pagos por aseguradoras) y factores externos (tal como organismos reguladores externos enfocados en el escrutinio de las prácticas y procesos para asegurar el control de calidad).

La práctica de hospitalismo se había reconocido ampliamente en Estados Unidos a fines de la década de 1980 y principios de 1990, pues muchos médicos comenzaron a dedicarse a practicar exclusivamente en el ámbito hospitalario - y definirse a sí mismos como hospitalistas-, acuñándose formalmente el término en 1996 por los doctores Robert Wachter y Lee Goldman en una publi- 
cación semanal en el New England Journal of Medicine. ${ }^{1}$ En ese entonces, surgieron modelos de atención médica que fueron conocidos como "atención administrada" o managed care. Esto imponía notables requerimientos burocráticos y de control para disminuir los costos, aunque mantenía la calidad de la atención médica, mejorando su eficiencia; también imponía barreras significativas, ya que para poder desempeñar una medicina de alto valor (definido por la calidad dividida sobre el costo), el médico debía concentrar su atención en un solo ámbito clínico (la consulta externa como médico de atención primaria, o bien como hospitalista).

Ya en la década de 1990, el enfoque de evitar la interrupción de los Servicios de Atención Ambulatoria, prevención de readmisiones y mejoría en la calidad de atención en la consulta externa derivó en que los médicos internistas de atención primaria prestaran atención intrahospitalaria a pacientes hospitalizados de menos de $10 \%$ de su tiempo. ${ }^{1}$ Al dedicarse exclusivamente a un solo ámbito, los primeros estudios sobre el modelo de medicina hospitalista se enfocaron en demostrar su impacto positivo en la disminución de los costos de atención médica, ${ }^{1,2}$ generando ahorros a los sistemas hospitalarios a través de la mejor utilización de recursos, disminución del tiempo de estancia hospitalaria y de readmisiones..$^{3-5}$

De manera interesante, el campo de la medicina hospitalista creció de manera exponencial, y tan sólo 20 años después, se ha convertido en un campo profesional muy fértil con más de 50 mil hospitalistas, quienes practican en más de $80 \%$ de los centros hospitalarios de Estados Unidos, y atienden a más de un tercio de pacientes del sistema de pago federal, conocido como Medicare. 6,7

La necesidad de contar con una sociedad profesional derivó en la creación de NAIP (National Association of Inpatient Physicians) por los doctores John R Nelson (Bellevue, Washington) y Winthrop F. Whitcomb (Springfield, Massachusetts) en 1997; ${ }^{6}$ en 2003, cambió su nombre a SHM (Society of Hospital Medicine), ${ }^{6}$ la cual es la casa académica y administrativa del movimiento hospitalista, y ésta tiene actualmente más de 17 mil miembros.

En el año 2000, el Instituto de Medicina publicó un libro seminal: To err is human (Errar es humano), el cual resaltó la presencia de errores médicos como causa relevante de mortalidad en Estados Unidos, atribuyendo 98 mil muertes anuales a esta causa. ${ }^{8}$ Esta visión reforzó la necesidad y papel del hospitalista en la mejora de la calidad de atención de salud y, aunado al acelerado crecimiento de la disciplina, llevó en 2006 a la publicación de las Competencias estructurales (o Core competencies) en Medicina Hospitalista. Esta publicación contiene los lineamientos curriculares de competencia profesional de los hospitalistas. ${ }^{9}$ En 2011, la American Board of Internal
Medicine implementó un examen enfocado a la práctica de medicina hospitalista. ${ }^{10,11}$ Hoy día, el médico hospitalista puede recertificarse por la vía tradicional o por el examen enfocado a la práctica hospitalista. ${ }^{11}$

Guiada por competencias curriculares muy precisas, ${ }^{8}$ la disciplina tuvo una evolución muy interesante, por ejemplo, ser los consultantes de Medicina Interna en pacientes quirúrgicos y desarrollar un servicio de manejo conjunto para esta población (co-management), demostrando una notable mejoría en la calidad de atención médica, que se caracteriza por una disminución en la tasa de complicaciones postquirúrgicas, menor necesidad de servicios consultantes, disminución en los costos intrahospitalarios, menor duración de estadía intrahospitalaria y menor tasa de readmisión. ${ }^{12}$

La necesidad de mantener el enfoque en el paciente hospitalizado se ha diversificado a múltiples disciplinas, tal como Pediatría (Medicina Hospitalista Pediátrica es reconocida como una subespecialidad de la Pediatría certificada desde el 2019 por el American Board of Pediatrics), ${ }^{13}$ Neurología, ${ }^{14}$ Ginecoobstetricia, ${ }^{6}$ Oncología, ${ }^{15}$ etcétera.

Entendiendo que es un eslabón más dentro del complejo sistema de salud, el médico hospitalista se ha convertido en un bastión de calidad y seguridad del paciente, y su desempeño profesional se ha extendido más allá de la práctica clínica, siendo comúnmente su participación como oficiales de calidad y seguridad del paciente, líderes de educación en los servicios académicos intrahospitalarios (por ejemplo, para estudiantes de medicina o residencias médicas), expertos en la mejoría de sistema de expediente electrónico, investigación clínica, administración hospitalaria, etcétera. ${ }^{6,7}$

A pesar de que la práctica del "hospitalismo" surgió desde las últimas décadas del siglo pasado, en México se conoce, pero se ejerce poco. Por ello, las instituciones educativas y sanitarias deberían incentivar la creación de programas universitarios para capacitar hospitalistas, de modo que puedan ser insertados en la práctica de la medicina institucional y privada, pues el beneficio para el ejercicio de la medicina en los hospitales sería muy grande y, tanto sociedad como sistemas de salud, se verían sumamente beneficiados.

\section{REFERENCIAS}

1. Wachter RM, Goldman L. The emerging role of "hospitalists" in the American health care system. N Engl J Med. 1996; 335: 514-517.

2. McMahon LF. The hospitalist movement-time to move on. N Engl J Med. 1996; 357 (25): 2627-2629.

3. Jungerwirth R, Wheeler SB, Paul JE. Association of hospitalist presence and hospital-level outcome measures among medicare patients. J Hosp Med. 2014; 9: 1-6. 
4. Auerbach AD, Wachter RM, Katz P, Showstack J, Baron RB, Goldman L. Implementation of a voluntary hospitalist service at a community teaching hospital: improved clinical efficiency and patient outcomes. Ann Intern Med. 2002; 137: 859-865.

5. Craig DE, Hartka L, Likosky WH, Caplan WM, Litsky P, Smithey J. Implementation of a hospitalist system in a large health maintenance organization: the Kaiser Permanente experience. Ann Intern Med. 1999; 130 (4 Pt 2): 355-359.

6. Messler J, Whitcomb W. A history of the hospitalist movement. Obstet Gynecol Clin N Am. 2015; 42: 419-432.

7. Wachter RM, Goldman L. Zero to 50,000-The 20th Anniversary of the hospitalist. N Engl J Med. 2016; 375 (11): 1009-1011.

8. Institute of Medicine (US) Committee on Quality of Health Care in America; Kohn LT, Corrigan JM, Donaldson MS, editors. To Err is Human: Building a Safer Health System. Washington (DC): National Academies Press (US); 2000. Available from: https://www.ncbi.nlm. nih.gov/books/NBK225182/. doi: 10.17226/9728
9. The core competencies in hospital medicine: A framework for curriculum development by the society of hospital medicine. J Hosp Med. 2006; 1: 2-95. doi: 10.1002/jhm.72.

10. Carris J. Focused practice in hospital medicine. The Hospitalist. 2010; 2010 (5).

11. ABIM Focuses Practice in Hospital Medicine Blueprint. https://www. abim.org/ /media/ABIM\%20Public/Files/pdf/exam-blueprints/ maintenance-of-certification/hospital-medicine.pdf

12. Rohatgi N, Loftus P, Grujic O, Cullen M, Hopkins J, Ahuja N. Surgical comanagement by hospitalists improves patient outcomes: a propensity score analysis. Ann Surg. 2016; 264: 275-282.

13. ABP Pediatric Hospital Medicine Certification. https://www.abp.org/ content/pediatric-hospital-medicine-certification

14. Barrett KM, Freeman WD. Emerging subspecialties in neurology: neurohospitalist. Neurology. 2010; 74 (2): e9-e10.

15. Beresford L. Oncology, surgical hospitalists most common as subspecialties gain followers. The Hospitalist. 2014; 2014 (12). 